\title{
Respiratory arrest: a complication of cerebellar ectopia in adults
}

\author{
D R FISH, * R S HOWARD, * C M WILES, * LINDSAY SIMON† \\ From the Department of Neurology, St Thomas' Hospital, ${ }^{*}$ and the Gough Cooper Department of Neurological \\ Surgery, $\uparrow$ The Institute of Neurology, London, UK
}

SUMMARY Two adult patients are described with cerebellar ectopia whose presentation at diagnosis was respiratory arrest. Following surgical decompression both patients become self ventilating and now lead independent lives. Cerebellar ectopia is a potentially remediable condition and should be considered in patients with unexplained respiratory arrest.

Arnold Chiari malformation type 1 (ACM I) usually presents in adults with occipital headaches, pain, weakness of the upper limbs, or unsteadiness of gait. ${ }^{2}$ Respiratory problems have been occasionally described in a number of reports. These have usually referred to postoperative abnormalities in ventilatory control, ${ }^{2-4}$ or infants with ACM II. ${ }^{56}$ We describe two adult patients with ACM I who suffered respiratory arrest.

\section{Case 1}

This 63 year old woman was admitted to hospital with severe respiratory failure, having been short of breath for the preceding 10 days. Thirteen years previously she had presented with a 6 year history of progressive difficulty in walking and a tendency to veer to the right. At that time she was noted to have a broad based gait and both horizontal and downbeat nystagmus. Plain skull radiographs showed minor basilar invagination, but myelography was normal. She remained stable until June 1985 when she presented with a 10 day history of severe occipital pain and breathlessness. There was a shorter history of difficulty in walking, weakness of the arms, dysphagia for solids and regurgitation of liquids. She was cyanosed, and had downbeat nystagmus, an absent gag reflex, and a spastic quadriparesis. Her chest radiograph was normal. She rapidly deteriorated and became unrousable, with shallow irregular respirations and apnoeic periods and required mechanical ventilation. Myelography showed changes suggestive of tonsillar herniation, and this was

Address for reprint requests: Dr D R Fish, The Institute of Neurology, Queen Square, London WCIN 3BG, UK

Received 17 July 1987 and in revised form 27 November 1987 Accepted 24 December 1987 confirmed on magnetic resonance imaging which also showed slight angulation of the medulla over the odontoid peg (fig, a). The fourth ventricle was normal. Exploration of the posterior fossa revealed low lying cerebellar tonsils thap came down well over the $\mathrm{Cl}$ segment and these were decompressed. She was weaned from the ventilator 2 weeks aftec the operation. Her respiratory pattern returned to normal and a sleep study showed no evidence of hypoventilation of apnoea. At the time of her discharge home she was able to ${ }^{+}$ walk with a frame, and swallow both liquids and solids without difficulty. She had remained well when reviewed a year later.

\section{Case 2}

This 54 year old woman was admitted to hospital in May 1982 having been found unconscious at home. She had been well until 1976 when she fell over while on holiday, and subsequently complained of difficulty in walking and swallowing. Her friends noted that she sometimes had long pauses between breaths. In 1982 she collapsed and was found to be unresponsive, apnoeic, and areflexic with extensor plantar responses. She required mechanical ventilation overnight but the next day she was alert and this was discontinued. However, over the next 3 weeks she had eight episodes of respiratory arrest during sleep, and subsequently became unable to breathe spontaneously for more than 30 minutes even when awake. She was transferred to the chronic respiratory unit at St Thomas' Hospital in February 1983. On examination at that time she was alert and orientated, but unable to stand. She was mildly dysarthric, and had downbeat nystagmus. Pinprick sensation was reduced in the right V1 and V2 and left V1 distribution, and the right sternomastoid was wasted. She had a spastic quadriparesis with bilateral extensor plantar responses, marked ataxia in all four limbs and impaired joint position sensation in the fingers of the right hand. Myelography and CT scan of the 

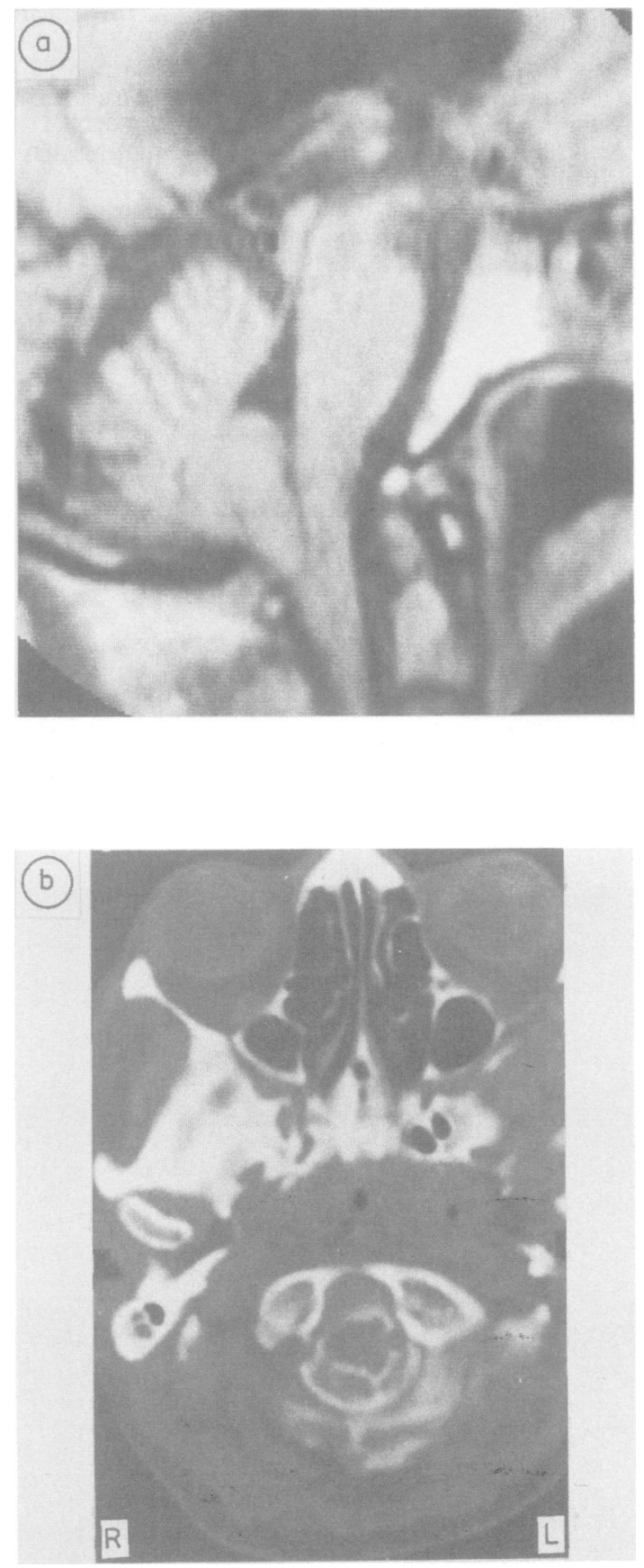

Fig (a) A sagittal section MRI through the posterior fossa and upper cervical cord of Case 1, showing elongation of the cerebellar tonsils through the foramen magnum down to the first cervical level. (b) Postmyelogram CT at the level of the occipital condyles of Case 2, showing cerebellar ectopia and distortion of the lower medulla. foramen magnum showed the cerebellar tonsils to extend below the arch of the atlas, with descent of the medulla into the upper cervical canal, and slight angulation over the odontoid peg (fig, $b$ ). The fourth ventricle was normal. Exploration of the posterior fossa revealed that both cerebellar tonsils were prolapsed, the left to the lower border of $\mathrm{Cl}$, and the right to the middle of $\mathrm{Cl}$, and these were decompressed. After operation she became self ventilating, her speech was less slurred, and she was able to swallow satisfactorily. She had a residual spastic quadriparesis and ataxia of all four limbs. Following rehabilitation she is able to live independently.

\section{Discussion}

Both the patients whom we report showed neurological abnormalities for many years before presentation, but the diagnosis of Arnold Chiari malformation was not made in either case. Presentation with progressive respiratory impairment, sleep apnoea ${ }^{7}$ or sudden respiratory deterioration due to obvious precipitating events ${ }^{8}$ has been described, but we have been unable to find any report of sudden unprovoked respiratory arrest in adults with previously undiagnosed ACM I.

In both patients the radiological and operative findings suggested compression of the brainstem and cord. This may have resulted in abnormal ventilatory control leading to hypoventilation and subsequent respiratory arrest.

The risk of sudden respiratory arrest as well as gradual respiratory deterioration, must be considered in planning the management of patients in whom a diagnosis of cerebellar ectopia has been made. Respiratory function testing and arterial blood gases should be routinely undertaken in these patients ${ }^{9}$ and, if nocturnal hypoventilation is suspected a sleep study should be performed. ${ }^{7}$ Furthermore, ACM I is a potentially treatable condition and should be considered in patients with unexplained respiratory arrest.

We are grateful to Dr G T Spencer, and Dr J H Marigold, St Thomas' Hospital, and Mr J F Shaw, Edinburgh Royal Infirmary, for permission to report cases under their care.

\section{References}

1 Spillane JD, Pallis C, Jones AM. Developmental abnormalities in the region of the foramen magnum. Brain 1957;80:11-48.

2 Mohr PD, Strang FA, Sambrook MA, Boddie HG. The clinical and surgical features in $\mathbf{4 0}$ patients with primary cerebellar ectopia (adult Chiari malformation). Q J Med 1977;181:85-96.

3 Krieger AJ. Respiratory failure as a surgical risk in 
patients with hindbrain anomalies. Heart Lung 1973;2:546-51.

4 Paul KS, Lye RH, Strang FA et al. Arnold Chiari malformation: A review of 71 cases. J Neurosurg 1983;58:183-7.

5 Papasomenos S, Roessmann U. Respiratory distress and the Arnold Chiari malformation. Neurology 1981;31:97-100.

6 Oren J, Kelly DH, Todres D, Shannon DC. Respiratory complications in patients with myelodysplasia and the
Arnold Chiari malformation. Am $J$ Dis Child 1986;140:221-4.

7 Balk RA, Hiller FC, Lucas EA, Scrina L, Wilson FJ, Wooten V. Sleep apnoea and the Arnold Chiari malformation. Am Rev Resp Dis 1985;132:929-30.

8 Smith J, Ridley A. Cerebellar ectopia presenting in adult life. Br Med J 1969;1:353-5.

9 Rosomoff HL. Occult respiratory and autonomic dysfunction in craniovertebral anomalies and upper cervical spine disease. Spine 1986;11:345-7. 